Benchmarks

\title{
The use of streptavidin conjugates as immunoblot loading controls and mitochondrial markers for use with Dictyostelium discoideum
}

\author{
Andrew J. Davidson", Jason S. King, , and Robert H. Insall \\ Beatson Institute for Cancer Research, Glasgow, UK \\ *A.J.D. and J.S.K. contributed equally to this work. \\ †J.S.'s current address is Department of Biomedical Science, University of Sheffield, Sheffield, UK. \\ BioTechniques 55:39-41 (July 2013) doi 10.2144/000114054 \\ Keywords: Dictyostelium; streptavidin; MCCC1; immunoblotting; mitochondria
}

Supplementary material for this article is available at www.BioTechniques.com/article/114054.

The loading controls used for quantitative immunoblotting of mammalian proteins are not appropriate for use with Dictyostelium discoideum. Actin levels, for example, change greatly during Dictyostelium development. In addition, Dictyosteliumspecific antibodies for other potential control proteins are not commercially available. Here we demonstrate the use of labeled streptavidin to detect biotinylated mitochondrial 3-methylcrotonyl-CoA carboxylase $\alpha$ (MCCC1), providing a robust and convenient tool for quantitative normalization of Dictyostelium Western blots, as well as fluorescently labeling mitochondria for microscopy of fixed cells.

Dictyostelium discoideum is a singlecelled, soil-dwelling amoeba that is a well established model organism used in the study of many cellular processes (1). However, a consistent problem that constrains Dictyostelium research is a dearth of loading controls suitable for use during immunoblotting. This is primarily due to a lack of commercially available antibodies, which severely impairs the ability of Dictyostelium researchers to perform quantitative studies of protein expression levels. Recently, Western blotting has advanced from traditional chemiluminescence based detection of one protein on one membrane, to multiplex detection of different proteins on the same membrane using fluorescently labeled secondary antibodies. This allows the expression levels of the protein of interest and a loading control to be measured on a single blot. As detection of both targets occurs on the same membrane, and the fluorescence signal is linear over a wider range of protein concentrations compared to chemiluminescence, this allows more precise quantification.

Antibody-epitope interactions are not the only high affinity and high specificity interactions that can support protein detection during immunoblotting. The interaction between streptavidin and biotin is one of the strongest known and it has been utilized for many different research purposes (2). Although a previous study suggested that Dictyostelium has as many as 20 endogenously biotinylated proteins (3), we found that probing immunoblots of Dictyostelium whole cell lysates with Alexa-680 conjugated streptavidin (Life Technologies, Paisley, UK) revealed only a single prominent band of approximately $80 \mathrm{kDa}$ (see Figure 1). Comparable results were also obtained with horseradish peroxidase (HRP) conjugated streptavidin (GE Healthcare, Amersham, UK) and traditional chemiluminescent detection (data not shown).

The single streptavidin-labeled band was extremely robust, and Dictyostelium cell pellets lysed with $150 \mathrm{mM} \mathrm{NaCl}, 10$ $\mathrm{mM}$ Tris pH 7.5, $1 \mathrm{mM}$ EGTA, $1 \mathrm{mM}$ EDTA and either 1\% NP40 (for SCAR blots) (4) or $0.1 \%$ SDS, $0.1 \%$ sodium deoxycholate, $50 \mathrm{mM}$ DTT, and HALT protease inhibitors (Thermo-Scientific, IL, USA) (for cAR1 blots) gave identical results (Figure 1). Protein levels were then determined using Precision Red Advanced Protein Assay Reagent (Cytoskeleton, Inc., CO, USA) before boiling for $5 \mathrm{~min}$ in LDS loading buffer (Life Technologies), except for cAR1 samples, which were not boiled. Samples were then run on $10 \%$ Bis/Tris NuPAGE polyacrylamide gels (Life Technologies) before transfer onto a nitrocellulose membrane and blocking with $5 \%$ milk at room temperature for $1 \mathrm{~h}$. When required, membranes were incubated in primary antibodies at $4^{\circ} \mathrm{C}$ overnight, followed by $3 \times 5 \mathrm{~min}$ washes in Tris-Buffered Saline (TBS, 50 $\mathrm{mM}$ Tris-Cl, pH 7.5, $150 \mathrm{mM} \mathrm{NaCl}$ ). Alexa 680-conjugated streptavidin was then combined at $20 \mathrm{ng} / \mathrm{mL}$ with the secondary antibody (Dylite 800-goat anti-rabbit, diluted 1:10,000 in TBS; Thermo-Scientific, IL, USA) and shaken with the membrane for $30 \mathrm{~min}$ at room temperature prior to $3 \times 5 \mathrm{~min}$ washes in TBST. Membranes were then imaged using a Li-Cor Odyssey fluorescence scanner (Lincoln, NE, USA).

Following streptavidin-agarose pull-down and mass spectrometry, the 80 $\mathrm{kDa}$ biotinylated protein was confirmed as 3-methylcrotonyl-CoA carboxylase $\alpha$ (MCCC1) (Dictybase ID: DDB G0287377), as previously predicted by others (3). In humans, MCCC1 is a wellcharacterized mitochondrial protein that is known to be heavily biotinylated $(5,6)$.

As we found that fluorescent- and HRP-conjugated streptavidin essentially only detect a single, strongly biotinylated

Method summary:

In Dictyostelium cell lysates, streptavidin conjugates only strongly label a single band on a Western blot, corresponding to a mitochondrial protein. Fluorescent or HRP-conjugated streptavidin can therefore be applied directly to either blots or fixed cells, providing a convenient loading control or a mitochondrial marker, respectively. 
A

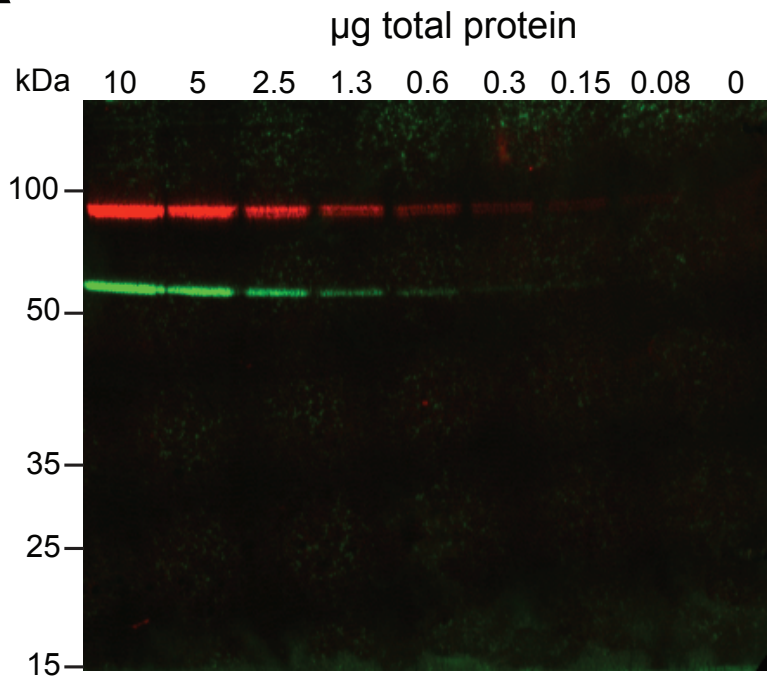

C

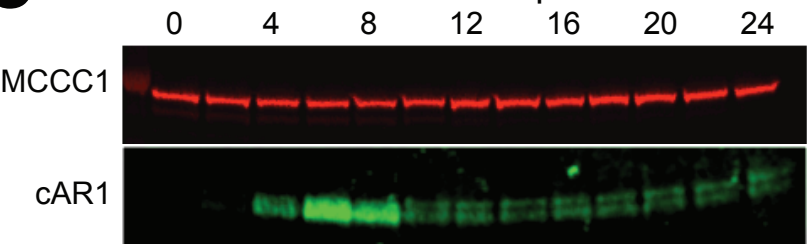

B

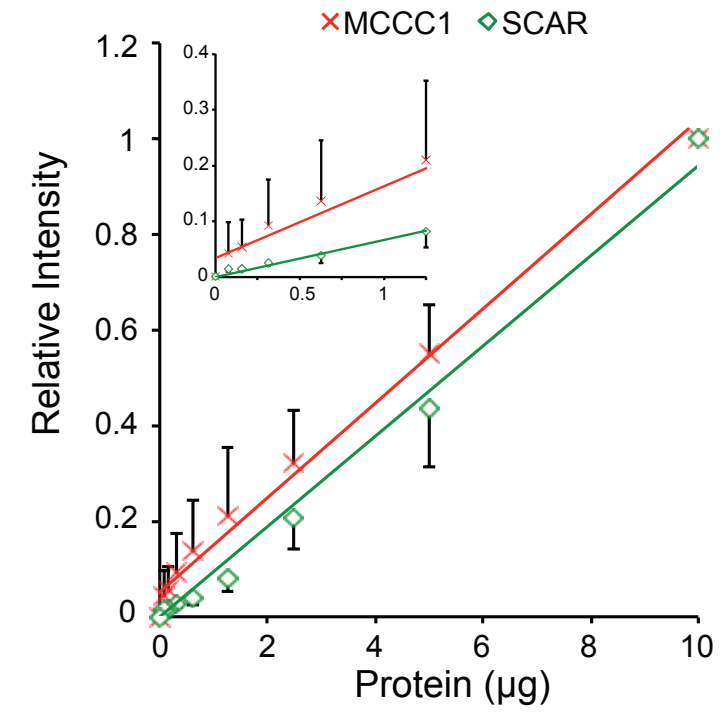

D

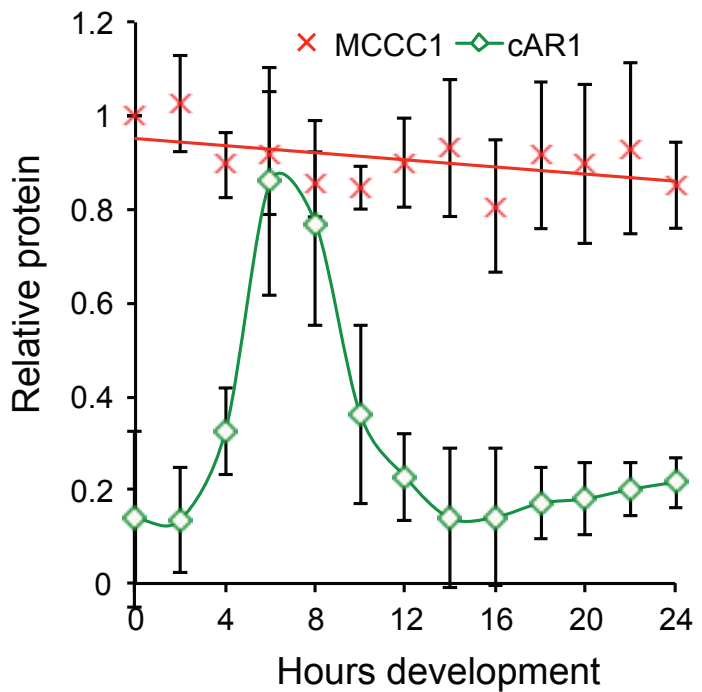

Figure 1. Quantitative immunoblotting of Dictyostelium whole cell lysates. (A) Whole cell lysates were serially diluted and the indicated amounts of total protein analyzed by Western blot. Membranes were probed simultaneously with both rabbit $\alpha$-SCAR antibody (green) and Alexa-680 streptavidin (red). (B) The intensity of each band was measured using ImageJ image analysis software (www.rsb.info.nih.gov/ij/), corrected for background fluorescence, and normalized to the highest concentration. The inset shows an enlargement of the values from $0-1.25 \mu g$ of protein. (C) Protein levels of both MCCC1 and cAR1 during Dictyostelium development. Samples were taken every $2 \mathrm{~h}$ throughout development, normalized for total protein content, and analyzed by Western blot. Membranes were then probed with both $\alpha$-cAR1 antibody (green) and Alexa-680 streptavidin (red). (D) Quantification of three independent experiments, with MCCC1 normalized to its initial level, and cAR1 normalized to its maximum levels. All values plotted are the mean \pm standard deviation of three experiments.

protein in Dictyostelium, we saw an opportunity to utilize MCCC1 as a loading control during immunoblotting. MCCC1 has many advantages for use as a loading control. It yields a single, strong clean band during blot imaging, and fluorescently tagged streptavidin can be used at very low concentrations. Because it can be applied to membranes directly without the requirement for a secondary antibody, streptavidin detection is extremely linear, especially when combined with fluores- cence. A variety of streptavidin conjugates are commercially available and inexpensive. Finally, streptavidin is not an antibody and therefore its use eliminates the inconvenience of cross-species antigenicity during dual color interrogation of the same membrane.

For use as an immunoblotting loading control, it is essential that the streptavidin/MCCC1 signal remains linear over a wide range of protein concentrations. We therefore analyzed serial dilutions of
Dictyostelium whole cell lysate by Western blot, probing the membrane with both Alexa-680 labeled streptavidin as well as a standard antibody (rabbit anti-SCAR primary antibody with Dylight 800 goat $\alpha$-rabbit secondary antibody) (7) on the same blot (Figure 1A and B). Importantly, the intensity of both the fluorescently labeled streptavidin and antibody-labeled bands remained linear over a $\sim 100$-fold range of protein concentration, validating the use of streptavidin/MCCC1 in combi- 

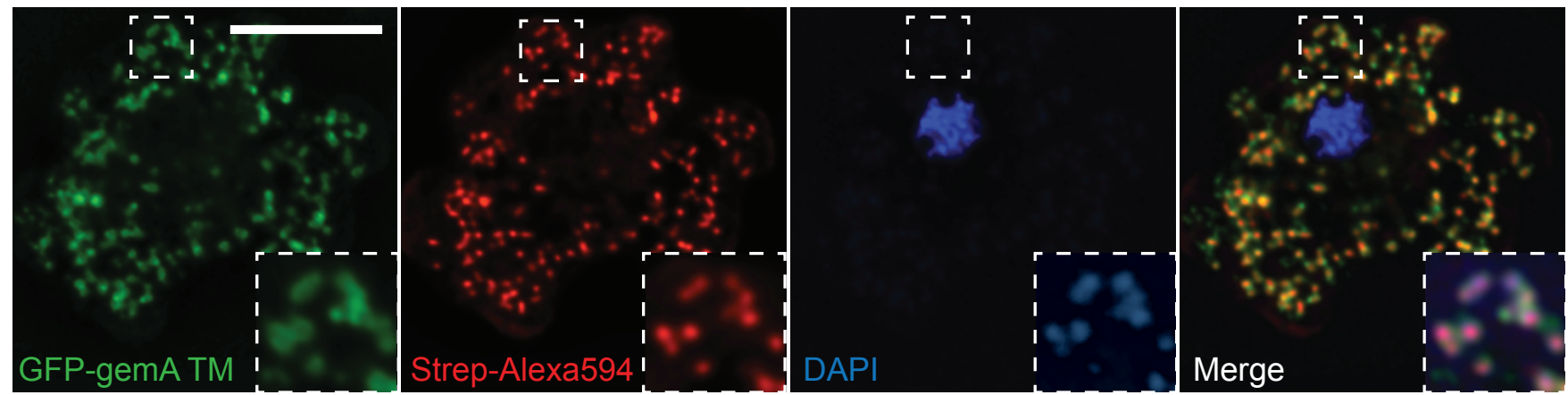

Figure 2. Co-localization of streptavidin conjugates with mitochondria. Ax3 cells expressing the mitochondrial outer membrane marker GFP-gemA TM were fixed in ultracold methanol and stained with Alexa-594 streptavidin and DAPI. A z-stack of widefield images was taken and deconvolved using the Volocity 6.2 software package (www.perkinelmer.co.uk/pages/020/cellularimaging/products/volocity.xhtml; Perkin Elmer Cambridge, UK). All three markers strongly co-localize, with the Alexa-594 streptavidin restrained within the lumen of the GFP and DAPI labeled mitochondria. The insets show a magnified region with the DAPI signal contrast enhanced to show the much dimmer mitochondrial signal. Bar represents $5 \mu$ m.

nation with conventional antibodies as a loading control for quantitative immunoblotting.

Dictyostelium has a well-defined developmental program that is the subject of many studies. However, protein expression during Dictyostelium development is notoriously variable due to a large decrease in cellular protein elicited by starvation, as well as massive transcriptional changes. It is therefore essential to have a reliable standard for normalizing the expression of developmentally regulated genes. The microarray and RNA-seq expression data available on the Dictybase community web site (www.dictybase.org/) both suggest that MCCC1 levels remain constant during the course of development. To confirm this, wild-type (Ax3) cells were plated for development on $1.5 \%$ agar coated Petri dishes and harvested every $2 \mathrm{~h}$ for $24 \mathrm{~h}$. Over this time course, the cells came together to form streams and then aggregates by approximately $6-10$ h of starvation. Multicellular slugs were observed around $14-16 \mathrm{~h}$ and fruiting bodies were seen by $24 \mathrm{~h}$.

Lysates were normalized to total protein content and analyzed by Western blot. MCCC1 and cAR1, a well characterized developmentally regulated gene (8), were detected using Alexa-680 labeled streptavidin and an anti-cAR 1 antibody (a kind gift from P. Devreotes). Consistent with previous studies, cAR1 expression peaked around $6-8 \mathrm{~h}$ of starvation (Figure $1 \mathrm{C}$ and D). In contrast, levels of MCCC1 protein remain constant throughout development, validating it as an appropriate control for developmental studies.

As MCCC1 is a mitochondrial protein, we also tested whether fluorescently conjugated strepatavidin could also be used as a mitochondrial marker. Cells expressing GFP fused to the transmembrane domain of gemA/Miro, which localizes to the mitochondrial outer membrane, (9) were fixed in ultracold methanol (10) and directly stained for $30 \mathrm{~min}$ with $20 \mathrm{ng}$ / $\mathrm{mL}$ Alexa-594 conjugated streptavidin (Life Technologies) and DAPI. Note that blocking was not required. As shown in Figure 2, the fluorescently labeled streptavidin strongly labeled the lumen of Dictyostelium mitochondria, co-localizing with the mitochondrial DAPI signal and surrounded by GFP-gemA TM. This demonstrates the use of fluorescently conjugated streptavidin as a convenient tool for staining mitochondria in fixed cells.

In conclusion, we have established the use of biotinylated MCCC1 as a convenient and robust control for quantitative immunoblotting or mitochondrial staining in Dictyostelium. Streptavidin conjugates can be applied to membranes or fixed cells directly, without the requirement for secondary antibodies, and used in combination with any antibody without crossreactivity. In addition to the applications demonstrated here, the range of streptavidin conjugates commercially available will allow their use as a convenient molecular tool for diverse additional applications.

\section{Acknowledgments}

We wish to thank Jane Borleis and Peter Devreotes for kindly providing us with the cAR1 antibody.

\section{Competing interests}

The authors declare no competing interests.

\section{References}

1. King, J.S. and R.H. Insall. 2009. Chemotaxis: finding the way forward with Dictyostelium. Trends Cell Biol. 19:523-530.

2. Weber, P.C., D.H. Ohlendorf, J.J. Wendoloski, and F.R. Salemme. 1989. Structural origins of high-affinity biotin binding to streptavidin. Science 243:85-88.

3. Lydan, M.A. and D.H. O'Day. 1991. Endogenous biotinylated proteins in Dictyostelium discoideum. Biochem. Biophys. Res. Commun. 174:990-994.

4. King, J.S., D.M. Veltman, M. Georgiou, B. Baum, and R.H. Insall. 2010. SCAR/WAVE is activated at mitosis and drives myosinindependent cytokinesis. J. Cell Sci. 123:22462255.

5. Apitz-Castro, R., K. Rehn, and F. Lynen. 1970. [Beta methylcrotonyl-CoA-carboxylase. Crystallization and some physical properties]. Eur. J. Biochem. 16:71-79.

6. Himes, R.H., D.L. Young, E. Ringelmann, and F. Lylen. 1963. The biochemical function of biotin. V. Further studies on beta-methylcrotonyl CoA carboxylase. Biochem. Z.337:48-61.

7. Blagg, S.L., M. Stewart, C. Sambles and R.H Insall. 2003. PIR121 regulates pseudopod dynamics and SCAR activity in Dictyostelium. Curr. Biol. 13:1480-1487.

8. Klein, P., R. Vaughan, J. Borleis, and P. Devreotes. 1987. The surface cyclic AMP receptor in Dictyostelium. Levels of ligandinduced phosphorylation, solubilization, identification of primary transcript, and developmental regulation of expression. J. Biol. Chem. 262:358-364.

9. Vlahou, G., M. Elias, J.C. von Kleist-Retzow, R.J. Wiesner, and F. Rivero. 2011. The Ras related GTPase Miro is not required for mitochondrial transport in Dictyostelium discoideum. Eur. J. Cell Biol. 90:342-355.

10. Hagedorn, M., E.M. Neuhaus, and T. Soldati. 2006. Optimized fixation and immunofluorescence staining methods for Dictyostelium cells. Methods Mol. Biol. 346:327-338.

Received 17 April 2013; accepted 15 May 2013.

Address correspondence to Jason S. King, Department of Biomedical Science, University of Sheffield, Sheffield, UK. E-mail: Jason.King@ Sheffield.ac.uk

To purchase reprints of this article, contact: biotechniques@fosterprinting.com 